\title{
Campomelic dysplasia: an overview of a rare genetic disorder
}

\author{
N. Antonakopoulos ${ }^{1}$, D. Vrachnis ${ }^{2}$, N. Loukas ${ }^{3}$, Ch. Christodoulaki ${ }^{4}$, Z. Iliodromiti ${ }^{5}$, N. Vrachnis ${ }^{1,3}$ \\ 'Gynecology, Obstetrics and Perinatal Medicine Unit, Evgenideio Hospital, National and Kapodistrian University \\ of Athens, Athens, Greece 2Endocrinology Unit, 2nd Department of Obstetrics and Gynecology, National and \\ Kapodistrian University of Athens Medical School, Aretaieio Hospital, Athens, Greece ${ }^{3} 3$ rd Department of \\ Obstetrics and Gynecology, National and Kapodistrian University of Athens Medical School, Attikon Hospital, \\ Athens, Greece ${ }^{4}$ Department of Obstetrics and Gynecology, Tzaneio Hospital, Peiraias, Greece ${ }^{5}$ Department of \\ Neonatology, National and Kapodistrian University of Athens Medical School, Aretaieio Hospital, Athens, Greece
}

Correspondence

N. Vrachnis MD, PhD, DFFP, PCME 124B Vas. Sophias av., Athens, 11526, Greece

\section{Abstract}

Campomelic dysplasia is a rare and severe genetic condition that is characterized by shortening and bowing of the long bones, abnormal face, multiple congenital anomalies, and ambiguous genitalia. Having conducted a review of the existing literature on this rare genetic disorder, we herein present the most pertinent and essential data on the condition viewed from the clinical perspective. In the majority of cases when the neonate survives the condition, since the underlying cause is more often than not a de novo mutation of the SOX9 gene, there is no increased risk of recurrence. Diagnosis is tentatively made based on skeletal findings during routine prenatal ultrasound; it may subsequently be confirmed via either prenatal or postnatal molecular genetic testing or else radiologic evaluation. In general, the condition is considered to be lethal in the neonatal period, there is no prenatal treatment and pregnancy termination is an option.

Key words: Campomelic dysplasia, SOX9, short and bowed long bones, ambiguous genitalia

\section{Introduction}

Skeletal dysplasias consist of a large heterogeneous group of more than 450 heritable disorders, such as achondroplasia, osteogenesis imperfecta, thanatophoric dysplasia, campomelic dysplasia and hypochondroplasia. The latter conditions are characterized by generalized abnormalities in cartilage and bone, primarily affecting the long bones, spine and head, which lead to abnormal skeleton shape and size. Typified by short stature, skeletal dysplasias may be accompanied by other system abnormalities, including neurologic, respiratory and cardiac. Campomelic dysplasia describes a form of skeletal dysplasia severely affecting development of the skeleton, the reproductive system, and other parts of the body. The term "campomelic" is derived from the Greek roots "campo" meaning "bent" and 
"melic" referring to a "limb". The estimated prevalence of the condition is between 1 in 40,000 and 1 in 200,000 individuals. The postnatal prognosis is guarded $^{1,2}$. For the present review, we searched the PubMed, Scopus, and Google Scholar databases in order to gather data on the cause and manifestations of the disease, as well as concerning prenatal diagnosis and management.

\section{Cause and manifestations}

The cause of the disease is mutations in the SOX9 gene $^{3}$, which contains instructions to make a specific protein that plays a crucial role, in the course of embryonic development, in the formation of a wide array of organs and tissues mainly concerning bones and cartilage. Moreover, the SOX9 protein additionally regulates the activity of yet other genes that are vital for the development of the skeleton and reproductive organs. In most cases of campomelic dysplasia, the mutation, which is de novo, is contained within the SOX9 gene and either inhibits the production of the SOX9 protein or results in a protein with impaired function. Approximately $5-10 \%$ of campomelic dysplasia cases are relatively mild forms that have been caused by chromosomal abnormalities occurring in the proximity of the SOX9 gene and thus disrupting regions of DNA which normally regulate SOX9 gene activity ${ }^{4,5,6}$.

Newborns affected with campomelic dysplasia are typically born with bowing and angular shape of the long bones of the legs and, occasionally, of the arms. Bowing can cause characteristic skin dimples, especially on the lower legs. Other features may be dislocated hips, 11 pairs of ribs instead of 12, underdeveloped shoulder blades, bone abnormalities of the neck, ambiguous genitalia, and clubfeet. Meanwhile, abnormal head and facial features can be macrocephaly, micrognathia, flat face, cleft palate, and prominent eyes ${ }^{1}$. Congenital laryngotracheomalacia (i.e., weakness and limpness of the walls of the trachea), which is often present in neonates with campomelic dysplasia, through causing serious breathing problems, contributes to the poor survival rate of these infants. Additional causes of neonatal death are respiratory failure secondary to airway compromise and cervical spine instability ${ }^{7}$. Neonates with campomelic dysplasia rarely survive past infancy ${ }^{8}$, although, given that the severity of the disease is variable, some do survive into adulthood. The latter individuals usually develop scoliosis and other spine abnormalities as they age, which may compress the spinal cord. Hearing loss is another late manifestation of the disease ${ }^{8}$.

\section{Prenatal diagnosis}

Skeletal findings on routine prenatal ultrasound lead to suspected diagnosis, and this can be confirmed via prenatal or postnatal molecular genetic testing or else radiologic evaluation. The routine anatomy scan will normally reveal ultrasound features, though certain milder forms may not be evident until later during the third trimester, particularly if only assessment of long bone length is carried out. Characteristic ultrasound findings are: bilateral bowed femurs and tibia (bilateral acute femoral angulation), bell-shaped, narrow chest, disproportionately small face and micrognathia (undersized jaw). Other possible sonographic findings are: increased nuchal translucency, large calvarium, hypoplastic scapulae, talipes and ambiguous genitalia ${ }^{9}$. Cardiac defects (Ventricular Septal Defect, Tetralogy of Fallot) are found in one-third of affected fetuses and hydronephronsis in a similar proportion ${ }^{2,10}$. A short femur length finding immediately rules out severe intrauterine growth restriction, which is normally associated with such features of placental insufficiency as oligohydramnios and abnormal Doppler indices. Therefore, the key sonographic sign is pronounced bowing of the long bones, while the anteroposterior plane of the bone where bowing occurs comprises the best plane to identify this feature. 


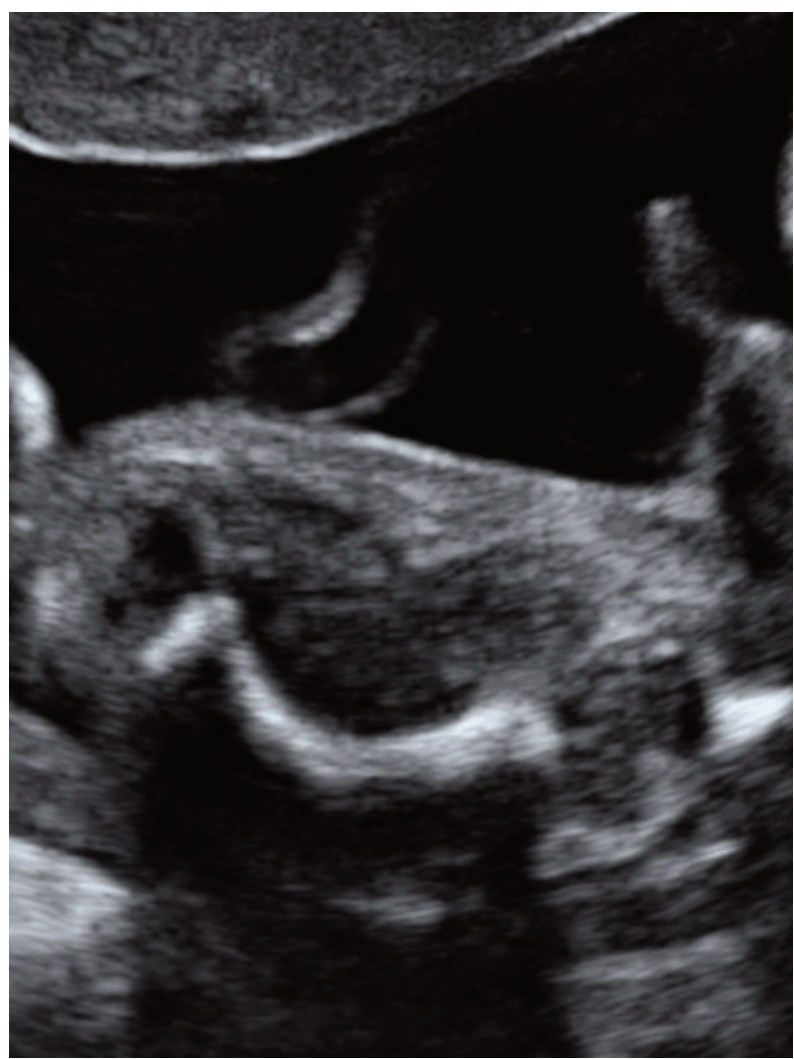

Figure 1. Ultrasound image of the short and bowed femur (arrow) of a fetus with Campomelic dysplasia at 24 weeks of gestation.

Prenatal diagnosis is possible with amniocentesis or chorionic villus sampling (CVS) for pregnancies at increased risk. Prior to performance of prenatal testing, there should be identification of the pathogenic allele of the affected family. Likewise, by performing prenatal amniocentesis or CVS, a diagnosis is possible in those pregnancies that have familial chromosome rearrangement. Another option for families who have an identified genetic cause is preimplantation genetic diagnosis ${ }^{11}$. Karyotype analysis is targeted to identify abnormalities involving the SOX9 locus on 17q24.3q25.1 and to clarify the genetic sex of the fetus $\mathrm{s}^{4,7}$.

\section{Management}

Prenatal counselling is problematic for most structural bone abnormalities. This is because it inevitably produces a differential or working diagnosis, with a precise diagnosis only being possible postnatally. Importantly, the outcome prediction necessitates the knowledge of a multidisciplinary team of fetal medicine specialists, neonatologists, geneticists, and orthopedic surgeons. Campomelic dysplasia is normally considered a lethal disorder, with pregnancy termination being an option, as there are to date no prenatal treatment options ${ }^{8}$.

However, if pregnancy continues, the issue of treatment, which is mainly palliative, symptomatic, and supportive, must be discussed with the parents. Follow-up and obstetric care are mandatory. Delivery should be conducted in a tertiary care center ${ }^{8}$.

On account of the long-term complications, children affected by campomelic dysplasia require constant follow-up by a team of specialists competent to deal with bone and joint problems, spine deformities, hearing loss, and cleft palate. In cases where the internal genitalia do not match the chromosomes' makeup, the gonads are sometimes removed to avoid an elevated risk of cancer later in life. The affected individuals need strong support to help them adjust and develop independence, while genetic counselling of the family will assess the risk of future children being affected and discuss alternatives.

\section{References}

1. S Unger, G Scherer, A Superti-Furga: Campomelic dysplasia. RA Pagon MP Adam HHArdinger et al. GeneReviews. 1993 Seattle (WA): GeneReviews.

2. The Fetal Medicine Foundation.

3. S McDowall, A Argentaro, S Ranganathan, et al.: Functional and structural studies of wild type SOX9 and mutations causing campomelic dysplasia. J Biol Chem. 1999; 274(34):24023-24030.

4. J Meyer, P Sudbeck, M Held, et al.: Mutational analysis of the SOX9 gene in campomelic dysplasia and autosomal sex reversal: lack of geno- 
type/phenotype correlations. Hum Mol Genet. 1997; 6 (1):91-98.

5. M Fukami, T Tsuchiya, S Takada, et al.: Complex genomic rearrangement in the SOX9 $5^{\prime}$ region in a patient with Pierre Robin sequence and hypoplastic left scapula. Am J Med Genet A. 2012; 158A (7):1529-1534.

6. G Kayhan, P Calis, D Karcaaltincaba, et al. Prenatal Diagnosis of campomelic dysplasia due to SOX9 deletion. J Obstet Gynaecol. 2019; 24:1-2.

7. SThomas, RB Winter, JE Lonstein: The treatment of progressive kyphoscoliosis in camptomelic dysplasia. Spine. 1997; 22 (12):1330-1337.

8. Joshua A. Copel, Mary E. D'Alton, Helen Feltovich, Eduard Gratacós, Deborah Krakow, Anthony O. Odibo, Lawrence D. Platt, Boris Tutschek. Obstetric imaging : fetal diagnosis and care. Second edition. | Philadelphia, PA : Elsevier, 2018.
9. L Zhen, LL Xu, DZ Li. Cystic hygroma and micromelic lower limbs: First-trimester sonographic markers of campomelic dysplasia. Eur J Obstet Gynecol Reprod Biol 2019; 238:191-193.

10. T Schramm, KP Gloning, S Minderer, et al.: Prenatal sonographic diagnosis of skeletal dysplasias. Ultrasound Obstet Gynecol. 2009; 34 (2):160170.

11. D. Higeta, R Yamaguchi, T Takagi, et al. Familial campomelic dysplasia due to maternal germinal mosaicism. Congenit Anom (Kyoto) 2018; 58 (6):194-197.

Received 10-4-2019

Revised 2-5-2019

Accepted 28-5-2019 